smoking and alcohol intake were not measured or indeed compared between the two groups. Furthermore, we are not given any indication as to how an individual was selected for scanning, as not all of the controls and patients recruited had a magnetic resonance imaging (MRI) scan. The authors did not use the same scanning techniques as Seidell et al (1990), who were among the first to describe the single-slice technique for estimating IAF area. There were large differences in terms of inversion and repetition times. Moreover, the most critical aspect of using a single scan to estimate IAF is to ensure that the scan is taken at the level of L4/L5 vertebra, which is best located by a radiological lateral scout and not palpation as performed by Zhang et al. Furthermore, MRI is not a 'precise and reliable means of determining the two fat measures with better resolution than computed tomography', as it can erroneously estimate the amount of IAF by $20 \%$.

From a statistical perspective, a oneway ANOVA should have been used to compare any differences between the three groups, as the use of multiple $t$-tests might have led to a type 1 error. A 'non-fasting glucose' level is not a standardised measure and is therefore meaningless. The actual values for fasting glucose decreased in both male and female patients, and fasting insulin levels decreased in females following treatment. Therefore, what Zhang et al show is that treatment with these two antipsychotics improves the metabolic profile of their patients despite an alleged increase in IAF.

Koro et al (2002) claim that olanzapine is associated with a higher risk of developing type 2 diabetes than risperidone, but this is difficult to interpret because Table 1 in their paper clearly indicates that the number of new cases of diabetes is greater in patients on risperidone $(5.1 \%)$ than olanzapine $(2.0 \%)$. There is little doubt that antipsychotics contribute to the development of type 2 diabetes in patients with schizophrenia. What is questionable is the magnitude of this effect. To date, the attributable risk for such an effect ranges between $2.03 \%$ for clozapine, $0.8 \%$ for quetiapine, $0.63 \%$ for olanzapine and $0.05 \%$ for risperidone (Leslie \& Rosenheck, 2004).

Despite the evidence presented the debate still centres on the diabetogenic effects of certain atypical antipsychotics. The purpose of the editorial was to put these issues into perspective to ensure that patients with schizophrenia, irrespective of their prescribed medication, would be offered screening for both diabetes and the metabolic syndrome.

Leslie, D. L. \& Rosenheck, R. A. (2004) Incidence of newly diagnosed diabetes attributable to atypical antipsychotic medications. American Journal of Psychiatry, 161, 1709-17|1.

Seidell, J. C., Bakker, C. J. \& van der Kooy, K. (1990) Imaging techniques for measuring adipose-tissue distribution - a comparison between computed tomography and 1.5-T magnetic resonance. American Journal of Clinical Nutrition, 5I, 953-957.

J. H.Thakore Neuroscience Centre, St Vincent's Hospital, Richmond Road, Dublin 3, Ireland. E-mail: j.thakore@rcsi.ie

\section{CBT for treatment-resistant schizophrenia}

We read with great interest the report on the randomised controlled trial (RCT) comparing cognitive-behavioural therapy (CBT) with supportive counselling for refractory psychotic symptoms of treatment-resistant schizophrenia (Valmaggia et al, 2005). It has a very convincing design but a few points need further discussion.

The sample size was calculated a priori, but an adequate number of patients could not be recruited. The small sample size led to a lack of statistical power, a limitation mentioned by the authors. However, this applied only to one intervention, supportive counselling, whereas there was an adequate estimated sample in the CBT group. Out of 62 participants randomised, post-treatment assessment was possible for 50 and followup was completed by 42 . Although sample attrition is understandable in this kind of study the withdrawal rate is relatively high. More people in the CBT group refused assessment post-treatment compared with those who received supportive counselling. The reason for this needs to be explained. Loss of data by the assessor, leading to exclusion from the intention-to-treat analysis was greater for the group who received supportive counselling; this group already had fewer participants and the loss of data might have influenced the result.

The treatment groups were not comparable at the beginning of the study for one illness variable. The supportive counselling group reported significantly more emotional distress related to auditory hallucinations. This is important because there was no difference between the groups post-treatment and at follow-up assessment. In addition, the changes in negative symptoms reportedly favoured supportive counselling.

Valmaggia et al stated that 'a larger percentage of participants in the cognitive-behavioural condition showed a $20 \%$ reduction in symptoms on the positive sub-scale of the PANSS' (Positive and Negative Syndrome Scale); however, comparative figures for both treatments and statistical significance would have illustrated this better.

Previous RCTs of the effect on symptoms of CBT compared with other psychological interventions showed a number needed to treat (NNT) of 5 (National Institute for Clinical Excellence, 2003). In the index study, the NNT was 3 but the confidence intervals were large in the two areas where a significant difference was measured for CBT.

Valmaggia et al stated that CBT for refractory psychotic symptoms of schizophrenia should be available in in-patient facilities. However, the evidence from their study is not unequivocal. Although the literature suggests benefits from psychological intervention in this group of patients, more robust evidence is still required to confidently recommend one particular type of therapy over others.

National Institute for Clinical Excellence (2003) Schizophrenia. Full National Clinical Guideline on Core Interventions in Primary and Secondary Care. London \& Leicester. Gaskell \& British Psychological Society.

Valmaggia, L. R., Van Der Gaag, M., Tarrier, N., et a (2005) Cognitive-behavioural therapy for refractory psychotic symptoms of schizophrenia resistant to atypical antipsychotic medication. Randomised controlled trial. British Journal of Psychiatry, 186, 324-330.

N. Kar, R. Dasi Wolverhampton City Primary Care Trust, Corner House Resource Centre, 300 Dunstall Road, Wolverhampton WV6 0NZ, UK. E-mail: nmadhab@yahoo.com

\section{Personality subtypes and cognitive impairment in anorexia nervosa}

I read with interest the article by Drs Thompson-Brenner and Westen (2005) about personality subtypes in eating disorders. Subnutrition from any cause is known to impair cognitive function and several workers have identified this in connection with anorexia nervosa (Macdonald, 1995).

The authors give no data on body mass index or weight. However, $38 \%$ of their sample had met criteria for anorexia nervosa at some point, $56 \%$ were fasting 4 days a week and half were exercising excessively. 
It is likely therefore that cognitive impairment was present in many. Hence the 'personality subtypes' identified may vanish on refeeding, or may be more pronounced, having been masked by cognitive blunting. It seems best to seek for personality subtypes in eating disorders during periods of adequate nutrition.

Macdonald, A. J. (1995) Eating disorders. Journal of Family Therapy, I7, 356

Thompson-Brenner, H. \& Westen, D. (2005) Personality subtypes in eating disorders: validation of a classification in a naturalistic sample. British Journal of Psychiatry, 186, 516-524.

A. J. Macdonald North Dorset Primary Care Trust, Forston Clinic, Dorchester DT2 9TB, UK. E-mail: ajmacdon@psychsft.freeserve.co.uk

Authors' reply: We appreciate Dr Macdonald's point that subnutrition may cause cognitive changes and other temporary conditions that may appear to affect personality. Data regarding personality in the context of adequate weight and nutrition are important for the accurate description of personality in eating disorders. It can be difficult, however, to ascertain whether shifts in personality functioning that take place through successful treatment or maturation precipitate positive nutritional changes or the reverse. Multiple studies do suggest that personality characteristics similar to those we describe in our report precede the development of eating disorders (Anderluh et al, 2003) and persist following remission of symptoms (Holtkamp et al, 2005). Although we did not report the data in detail, only $6.9 \%$ of those in our sample had a weight $15 \%$ below ideal, and the number of days of fasting was not correlated with either measure of personality pathology, suggesting this issue may not have compromised our data regarding personality to a significant extent.

\section{Anderluh, M. B., Tchanturia, K., Rabe-Hesketh, S.} et al (2003) Childhood obsessive-compulsive personality traits in adult women with eating disorders: defining a broader eating disorder phenotype. American Journal of Psychiatry, 160, 242-247.

Holtkamp, K., Muller, B., Heussen, N., et al (2005) Depression, anxiety, and obsessionality in long-term recovered patients with adolescent-onset anorexia nervosa. European Child and Adolescent Psychiatry, I4, $106-110$

H.Thompson-Brenner, D. Westen Eating Disorders Program, Center for Anxiety and Related Disorders, Psychology Department, Boston
University, 648 Beacon Street, Boston, MA 02215, USA. E-mail: htl4l@hotmail.com

\section{Psychedelics in psychiatry}

In his editorial 'Can psychedelics have a role in psychiatry once again?' (Sessa, 2005), Dr Sessa offers a detailed historical and heuristic perspective of psychedelics, with particular reference to psychotherapy. Reading the article the feeling was of sensed (by the author) repulsion of the 'neurobiological' psychiatrist in relation to 'research that explores alternative states of consciousness', 'psychedelics research' as a 'viable neurobiologial substrate for the very human experience of religious encounter' and generally a possible use of psychedelics in psychiatry. Perhaps we are some of those psychiatrists who 'have been conditioned to consider such work as mysticism' but we found such a proposition challenging. We would like to discuss recent neurobiological findings related to one of the psychedelics mentioned by Dr Sessa, which perhaps would offer an explanation as to why these substances have limited scope in psychiatry today.

3,4-Methylenedioxymethamphetamine (MDMA), also known as ecstasy, is largely consumed by young adults as a recreational drug. Common doses of this popular compound $(60-120 \mathrm{mg}$, equivalent to $1-$ 2 tablets) produce unexpectedly high blood levels, with MDMA present at high concentration at the receptor level. The drug induces dose-dependent neurotoxicity in animal models and humans; this mainly involves the central serotonergic system (Ricaurte et al, 2002). Serotonin is important for brain development and maintenance of neural and glial function in the mature brain (Azmitia, 2001). Another interesting mechanism involves the 'pruning' of serotoninergic neurons (Ricaurte et al, 2000). The drug appears to reduce the number of serotonin axons and axon terminals but nerve cells will often replace terminals upstream for the damaged ones. The resulting effect is of substantial impaired connectivity. Younger brains are particularly susceptible because of increased neuroplasticity, resulting in a substantial reorganisation of brain connectivity.

Functional magnetic resonance imaging studies suggest a decreased activation in inferior temporal regions, the hippocampus, angular gyrus and striate cortex associated with working memory performance (Daumann et al, 2003, 2005), with the hippocampus and globus pallidus being possibly more sensitive (Reneman et al, 2001; Jacobsen et al, 2004; Daumann et al, 2005). More recent voxel-based morphometry studies support the hypothesis that the use of MDMA leads to reduction in cortical grey matter in multiple brain regions, including the neocortex, brain-stem, cerebellum and anterior cingulate gyrus, reflecting compromised serotonergic activity (Cowan et al, 2003). Although we have mentioned only a few studies, there is substantial evidence to suggest considerable neurotoxicity of compounds such as those mentioned by $\mathrm{Dr}$ Sessa. There are concerns about possible long-term adverse effects of psychedelics in both infrequent and regular users, which explain why psychiatrists are reluctant to consider such substances in their pharmacological armamentarium.

Azmitia, E. C. (200I) Modern views on an ancient chemical: serotonin effects on cell proliferation, maturation, and apoptosis. Brain Research Bulletin, 56. $413-424$.

Cowan, R. L., Lyoo, I. K., Sung, S. M., et al (2003) Reduced cortical gray matter density in human MDMA (Ecstasy) users: a voxel-based morphometry study. Drug and Alcohol Dependence, 72, 225-235.

Daumann, J., Schnitker, R., Weidermann, J., et al (2003) Neural correlates of working memory in pure and polyvalent ecstasy (MDMA) users. Neuroreport, 14 1983-1987.

Daumann, J., Fischermann, T., Heekeren, K., et a (2005) Memory-related hippocampal dysfunction in poly-drug ecstasy (3,4-methylenedioxymethamphetamine) users. Psychopharmacology, 180, 607-6II.

Jacobsen, J. K., Mencl, W. E., Pugh, K. R., et al (2004)

Preliminary evidence of hippocampal dysfunction in adolescent MDMA ('ecstasy') users: possible relationship to neurotoxic effects. Psychopharmacology, 173, 383-390.

Reneman, L., Majoie, C. B., Habraken, J. B., et a (200I) Effects of ecstasy (MDMA) on the brain in abstinent users: initial observations with diffusion and perfusion MR imaging. Radiology, 220, 611-617.

Ricaurte, G. A., Yuan, J., McCann, U. D. (2000) ( \pm )3,4-Methylenedioxymethamphetamine ('Ecstasy')induced serotonin neurotoxicity: studies in animals. Neuropsychobiology, 42, 5-10.

Ricaurte, G. A., Yuan, J., Hatzidimitriou, G., et a (2002) Severe dopaminergic neurotoxicity in primates after a common recreational dose regimen of MDMA ('ecstasy'). Science, 297, 2260-2263.

Sessa, B. (2005) Can psychedelics have a role in psychiatry once again? British Journal of Psychiatry, $\mathbf{1 8 6}$ $457-458$.

D. Arnone, F. Schifano Department of Menta Health - Addictive Behaviour, St George's University of London, Cranmer Terrace, London SWI7 ORE, UK. E-mail: darnone@sgul.ac.uk 